\title{
Proteomic Signature of Nucleus Pulposus in Fetal Intervertebral Disc
}

\author{
Shanmuganathan Rajasekaran ${ }^{1}$, Dilip Chand Raja Soundararajan ${ }^{1}$, Chitraa Tangavel ${ }^{2}$, Sri Vijay Anand K. S. ${ }^{1}$, \\ Sharon Miracle Nayagam ${ }^{2}$, Monica Steffi Matchado ${ }^{2}$, Raveendran Muthurajan ${ }^{3}$, Ajoy Prasad Shetty ${ }^{1}$, \\ Rishi Mugesh Kanna ${ }^{1}$, K. Dharmalingam ${ }^{4}$ \\ ${ }^{1}$ Department of Spine Surgery, Ganga Hospital, Coimbatore, India \\ ${ }^{2}$ Ganga Research Centre, Coimbatore, India \\ ${ }^{3}$ Department of Plant Biotechnology, Tamil Nadu Agricultural University, Coimbatore, India \\ ${ }^{4}$ Aravind Medical Research Foundation, Madurai, India
}

\begin{abstract}
Study Design: Profiling proteins expressed in the nucleus pulposus of fetal intervertebral disc (IVD).
Purpose: To evaluate the molecular complexity of fetal IVDs not exposed to mechanical, traumatic, inflammatory, or infective insults to generate improved knowledge on disc homeostasis.

Overview of Literature: Low back pain is the most common musculoskeletal disorder, causing a significant reduction in the quality of life, and degenerative disc disorders mainly contribute to the increasing socioeconomic burden. Despite extensive research, the causative pathomechanisms behind degenerative disc disorders are poorly understood. Precise molecular studies on the intricate biological processes involved in maintaining normal disc homeostasis are needed.

Methods: IVDs of nine fetal specimens obtained from medical abortions were used to dissect out the annulus fibrosus and nucleus pulposus under sterile operating conditions. Dissected tissues were transferred to sterile Cryovials and snap frozen in liquid nitrogen before transporting to the research laboratory for protein extraction and further liquid chromatography tandem mass spectrometry (LCMS/MS) analysis. Collected data were further analyzed using Gene Functional Classification Tool in DAVID and STRING databases. Results: A total of 1,316 proteins were identified through LC-MS/MS analysis of nine fetal IVD tissues. Approximately 247 proteins present in at least four fetal discs were subjected to further bioinformatic analysis. The following 10 clusters of proteins were identified: collagens, ribosomal proteins, small leucine-rich proteins, matrilin and thrombospondin, annexins, protein disulfide isomerase family proteins and peroxiredoxins, tubulins, histones, hemoglobin, and prolyl 4-hydroxylase family proteins.

Conclusions: This study provides fundamental information on the proteome networks involved in the growth and development of healthy fetal discs in humans. Systematic cataloging of proteins involved in various structural and regulatory processes has been performed. Proteins expressed most abundantly (collagen type XIV alpha 1 chain, biglycan, matrilin 1, and thrombospondin 1) in their respective clusters also elucidate the possibility of utilizing these proteins for potential regenerative therapies.
\end{abstract}

Keywords: Intervertebral disc degeneration; Low back pain; Disc degeneration; Fetal intervertebral disc proteome

This is the 2019 APSS-Asian Spine Journal Best Paper Award.

Received Jul 2, 2019; Revised Sep 2, 2019; Accepted Sep 19, 2019

Corresponding author: Shanmuganathan Rajasekaran

Department of Spine Surgery, Ganga Hospital, 313, Mettupalayam Road, Coimbatore-641043, Tamil Nadu, India

Tel: +91-9843022325, Fax: +91-422-4383863, E-mail: rajasekaran.orth@gmail.com 


\section{Introduction}

Intervertebral disc (IVD) degeneration leading to low back pain is rapidly becoming a common musculoskeletal disorder, posing serious threats in terms of loss of productivity and increase in health expenditure toward diagnosis and treatment. The current diagnostic methods are proved to be sensitive in the diagnosis of disc degeneration only during its later stages of development; hence, surgical interventions are becoming unavoidable to mechanically resolve the pain. In this regard, development of therapeutic procedures for the restoration of the physiological process in the disc will help avoid surgical interventions, but it needs greater understanding of the molecular homeostasis of the discs and molecular processes affected during degeneration. Several attempts have been made to resolve the molecular complexity during disc degeneration by comparing degenerated discs against relatively normal scoliotic/traumatic discs. No attempts have been made to profile the gene expression in absolutely normal discs due to several difficulties. A recent review discusses the embryonic development of IVD in mouse models as it is difficult to elucidate the same in human embryos due to ethical reasons [1]. In this study, attempts were made to catalog the proteome of developing IVDs of the human fetus, which elucidates the molecular networks regulating the growth and development of IVD and have opened avenues for developing tools and kits for early diagnosis and noninvasive therapeutic treatment of degenerative disc disorder (DDD).

\section{Materials and Methods}

\section{Sample collection}

Nine fetal specimens were collected following spontaneous and medical abortions (24 weeks of gestational age) upon proper approval from the Institutional Ethical Committee and Institutional Review Board (Ganga Medical Centre and Hospitals Pvt Ltd., approval no., 8/12/2018). Six cases of abortion were spontaneous, whereas three were medically terminated pregnancies caused by the following: (1) cleft lip and palate with severe facial anomaly, (2) uncontrolled gestational diabetes mellitus causing diabetic nephropathy to the mother and oligohydramnios to the fetus, and (3) unplanned pregnancy in a complete family of five children. All fetal specimens were screened for spinal diseases and devoid of spine pathologies, such as congenital anomalies and open neural tube defects. After obtaining informed consent from the parents of the deceased fetus, the spine segment was harvested through a posterior approach within 12 hours of death under sterile operating conditions. Fetuses with normal magnetic resonance imaging and computed tomography findings were included, and those with skeletal disorders and proven syndromic features were excluded from the study. IVD tissues were dissected out from the samples and separated into the nucleus pulposus (NP) and annulus fibrosus using a surgical microscope (Fig. 1). Then, NP tissues were washed in phosphate buffer solution and stored in sterile Cryovials, which were then placed in liquid nitrogen for further research.

\section{Protein extraction, purification, and peptide digestion}

Frozen tissues were thawed on ice before pulverization using liquid nitrogen $\left(-196^{\circ} \mathrm{C}\right)$. Approximately $100 \mathrm{mg}$ tissue from the nine fetal discs was subjected to extraction of total proteins. The weighed tissues were homogenized using radioimmunoprecipitation assay (RIPA) buffer. Following initial homogenization, the pellets were further subjected to extraction using $2 \%$ sodium dodecyl sulfate (SDS) buffer to facilitate extraction of the entire protein content. In the extraction using RIPA buffer, soluble fraction yielded hydrophilic proteins, whereas insoluble fraction using $2 \%$ SDS buffer yielded mostly hydrophobic proteins.

The extracted total proteins were subjected to methanolchloroform precipitation method to remove salts, detergents, and other interfering agents in the following ratio: 1 part of sample:4 parts of methanol:1 part of chloroform:3 parts of water. The resulting protein pellet was resuspended in $1 \times$ Laemmli buffer for SDS-polyacrylamide gel electrophoresis (PAGE) analysis.

Protein concentrations in clean samples were estimated using bicinchoninic acid assay. Approximately 50-100 $\mu \mathrm{g}$ of protein sample was loaded on $12 \%$ SDS-PAGE for protein separation, and further protein bands were developed using Coomassie Brilliant Blue G-250 stain. The entire lane of interest was excised into separate bands and chopped into 150-300 $\mu \mathrm{m}$ small pieces prior to reduction with dithiothreitol, alkylation with iodoacetic acid, and enzymatic digestion with trypsin for 12-16 hours of incubation at $37^{\circ} \mathrm{C}$. 

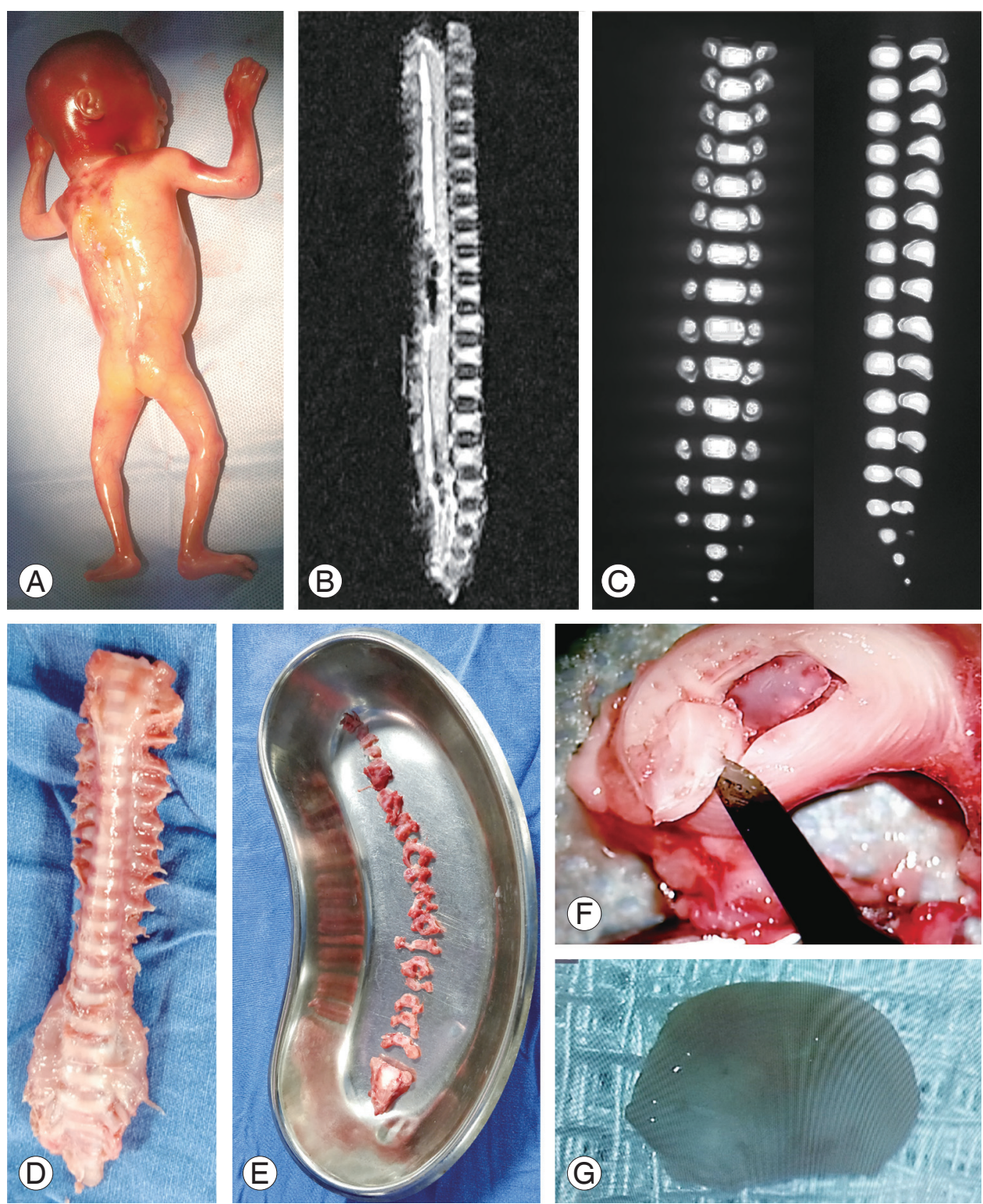

Fig. 1. (A) Fetal specimens obtained after medical abortion for research with due consent from parents. (B) Magnetic resonance imaging and (C) computed tomography showing well-formed normal spine segments. (D) Whole spine segment harvested from the fetus through a posterior approach. (E) Vertebral bone separated from (F) disc tissues that were further dissected under the microscope to separate the $(\mathbf{G})$ nucleus pulposus tissue for proteomic analysis.

Tryptic peptides were purified using Agilent spin columns (Cat no., 5188-2750). Purified samples were dried under vacuum conditions. They were then resuspended in $0.1 \%$ formic acid before loading in Orbitrap Velos Pro mass spectrometer for identification (Fig. 2).

\section{Liquid chromatography tandem mass spectrometry analysis}

Purified trypsin-digested peptides were analyzed by nano- reverse-phase liquid chromatography (LC)-tandem mass spectrometry (MS/MS) using a Thermo Easy-nLC 1000 (Thermo Fisher Scientific, Waltham, MA, USA) coupled with Orbitrap Velos Pro mass spectrometer (Thermo Fisher Scientific). The experimental conditions are presented in Table 1. The linear trap quadrupole was calibrated using the positive ion calibrant solution (Cat no., 88323, Pierce) and tuned to optimize the response of the ion at $\mathrm{m} / \mathrm{z} 524$ of the tetra peptide Met-Arg-Phe-Ala MS/ MS. 


\section{Bioinformatics analysis}

Raw data obtained through LC-MS/MS were analyzed by Proteome Discoverer version 1.4 (Thermo Fisher Scientific) using Mascot software version 2.4.1.0 (Matrix Science, London, UK) and SequestHT search algorithm. The peptide spectrum matches from SequestHT and Mascot were post-processed using the Percolator algorithm. Peptides with a $q$-value lesser than the threshold of 0.01 were considered for protein identification. The best match is given rank 1 . The resultant search data from all bands for each sample were combined to generate a multi-consensus re-

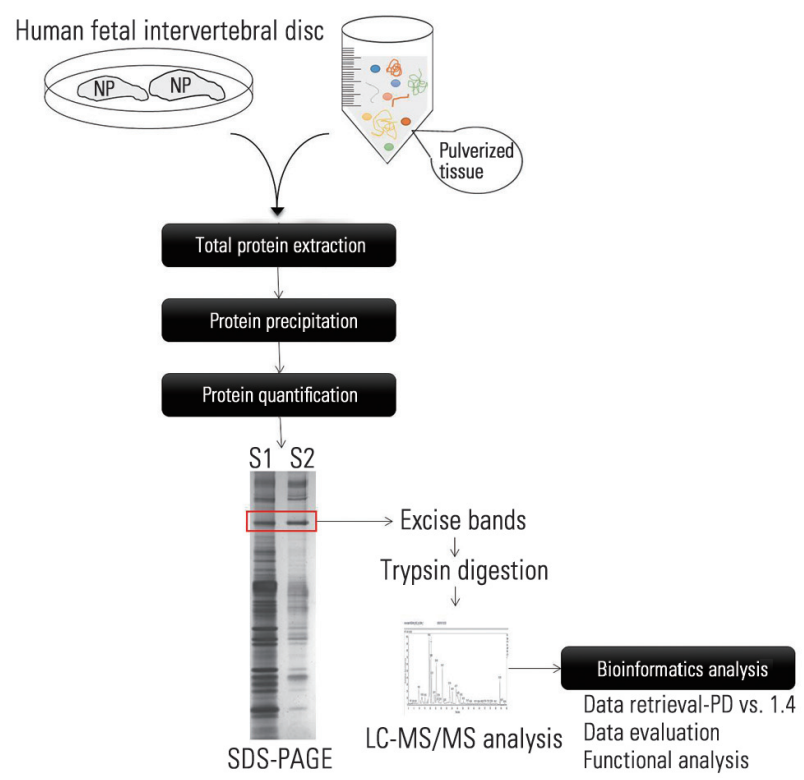

Fig. 2. General workflow of protein extraction and identification. S1, soluble fraction; S2, insoluble fraction; SDR-PAGE, sodium dodecyl sulphate polyacrylamide gel electrophoresis; LC, liquid chromatography; MS/MS, tandem mass spectrometry; PD, proteome discoverer. port to obtain a non-redundant list of identified proteins. This list was further filtered with high peptide confidence and peptide rank 1 to obtain reliable identification results for further data interpretation.

\section{Relative quantification by spectral count}

Spectral counts obtained by LC/MS-MS analysis were further normalized by normalized spectral abundance factor method [2].

\section{Gene ontology and pathway enrichment analysis}

Gene ontology (GO) and pathway term enrichment was performed to catalog the nature of fetal proteome in terms of biological process, molecular function, cellular component, and pathways using clusterProfiler, ReactomePA R packages (https://www.bioconductor.org/), and STRING (https://string-db.org/). This analysis helped determine the more frequently occurring pathways and GO terms than by chance.

\section{Functional clustering of fetal proteome}

Proteins with high confidence were clustered based on functional similarity using the DAVID Gene Functional Classification Tool. The tool utilizes the novel heuristic multiple linkage clustering to measure functional enrichment in annotation categories of 75,000 terms from 14 functional annotation sources. Group enrichment score ranks protein clusters based on the overall $p$-value of all enriched annotations.

Table 1. Details of experimental conditions in the LC-MS/MS analysis

\begin{tabular}{|c|c|c|}
\hline Instrument & Specification & Settings \\
\hline LC-precolumn & $\begin{array}{l}\text { Acclaim PepMap 100, C18, } 3 \mu \mathrm{m} \text { particle size, } \\
100 \AA, 75 \mu \mathrm{m} \times 2 \mathrm{~cm}\end{array}$ & Maximum pressure 700 bar \\
\hline LC-capillary column & $\begin{array}{l}\text { EASY-spray column PepMap RSLC, C18, } 2 \mu \mathrm{m} \text {, } \\
100 \AA, 75 \mu \mathrm{m} \times 50 \mathrm{~cm} \text { or } 15 \mathrm{~cm}\end{array}$ & $\begin{array}{l}\text { Linear gradient: } 5 \% \text { B to } 100 \% \text { B over } 90 \mathrm{~min} \text { at a constant flow } \\
\text { rate of } 200 \mathrm{~nL} / \mathrm{min}, 95 \% \text { acetonitrile in } 0.1 \% \text { formic acid from } \\
5 \% \text { to } 30 \% \text { over } 72 \mathrm{~min}, 30 \% \text { to } 100 \% \text { over } 10 \mathrm{~min} \text {, and kept } \\
\text { at } 100 \% \text { for } 5 \mathrm{~min} \text { at a flow rate of } 200 \mathrm{~nL} / \mathrm{min}\end{array}$ \\
\hline \multirow[t]{4}{*}{ MS/MS: positive mode electrospray ionization } & lon spray voltage & $2.4 \mathrm{kV}$ \\
\hline & Capillary temperature & $200^{\circ} \mathrm{C}$ \\
\hline & Radio frequency lens voltage & 69 \\
\hline & Maximum injection time & $50 \mathrm{~ms}$ \\
\hline
\end{tabular}

LC, liquid chromatography; MS/MS, tandem mass spectrometry. 


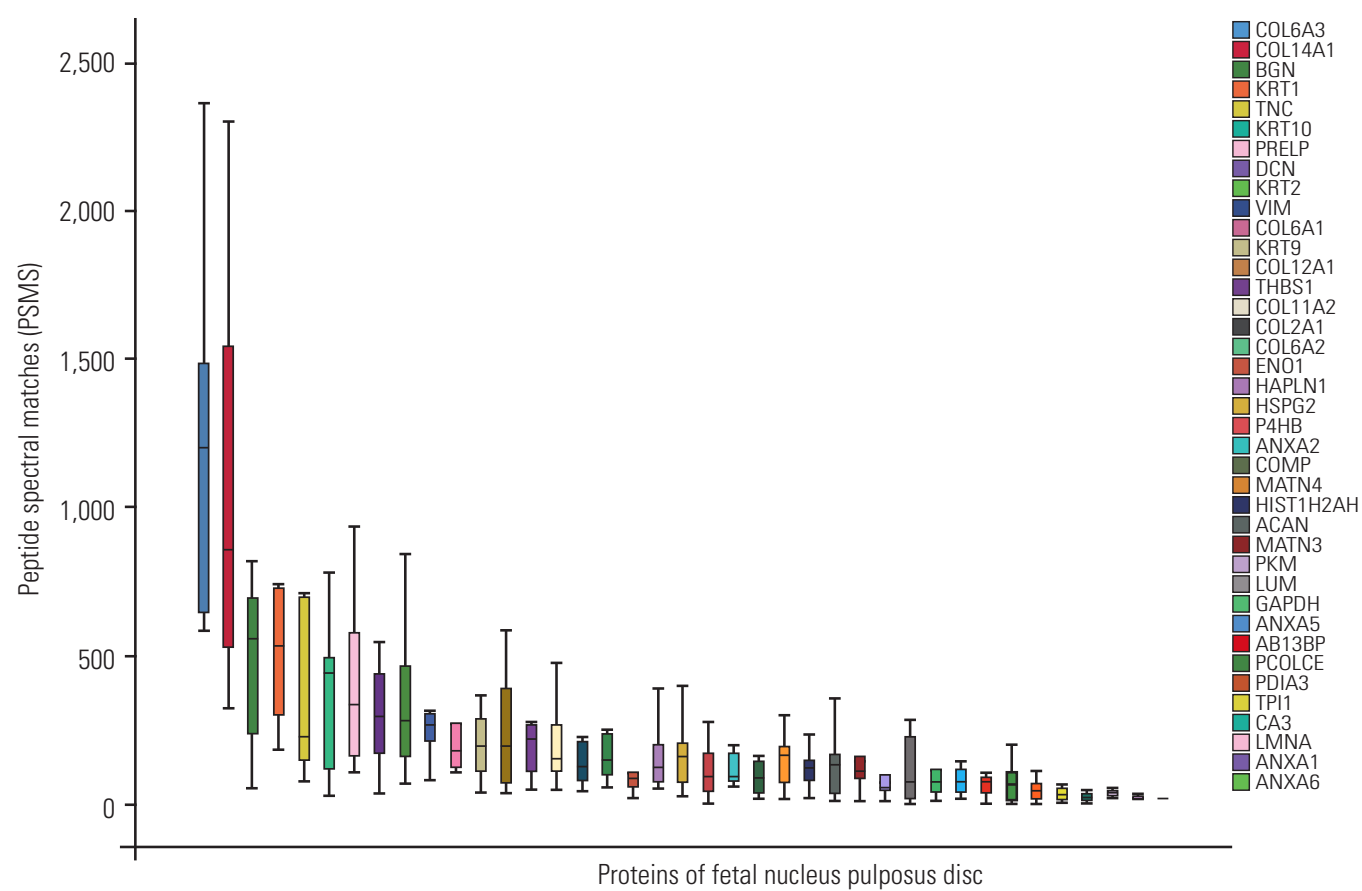

Fig. 3. Expression of most abundant proteins identified among the nine fetal discs.

\section{Results}

LC-MS/MS analysis of nine fetal discs identified 1,316 proteins. Proteins and their peptide sequences were verified with the manually curated SWISS-PROT database to remove unreviewed protein entries. Uncharacterized or putative or fragment proteins were excluded in the further analysis and are separately presented in Supplemental Table 1.

\section{Frequency of protein detection}

Approximately $20 \%$ of proteins identified in the fetal disc were similar to adult disc samples identified in our earlier studies. Extracellular matrix and structural constituents conferring tensile strength to the discs, such as collagens, proteoglycans, glycoproteins, and annexins, were present in all nine samples. Other frequently detected proteins include small leucine-rich proteins (SLRPs), clusterin, matrilin, ribosomal proteins, histidines, globin family, tubulins, keratins, and peroxiredoxins, as shown in Fig. 3 and Supplemental Table 1. Approximately 173 proteins were found in four to seven samples, whereas approximately $60 \%$ of proteins were found in any one of the samples that are low abundant, mainly participating in adenosine triphosphate hydrolysis and metabolic, cellular, catabolic, and nucleotide binding processes.
To understand the matrisomal profiling of fetal NP discs, proteins were filtered based on the matrisome database (http://matrisome.org). Quantitative analysis was performed based on the peptide spectral match counts of total identified proteins. It revealed that $48 \%$ of total proteins contributed to matrisomal proteins and $52 \%$ were other proteins.

Matrisomal proteins were further divided into two major categories: core matrisome (63\%) and matrisomeassociated proteins (37\%). Core matrisomes were composed of proteoglycans (20\%), collagens (20\%), and glycoproteins (56\%). Matrisome-associated proteins were also subcategorized into matrisome-affiliated proteins (67\%), regulators (30\%), and secreted factors (3\%).

\section{Gene ontology}

Significant proteins were considered for the GO enrichment analysis. The distribution of biological processes, molecular function, and cellular component was obtained using clusterProfiler package. The distribution of biological process and cellular component across the IVD are shown in Fig. 4 along their $p$-values.

\section{Cluster analysis}

Approximately 247 proteins with high confidence were 


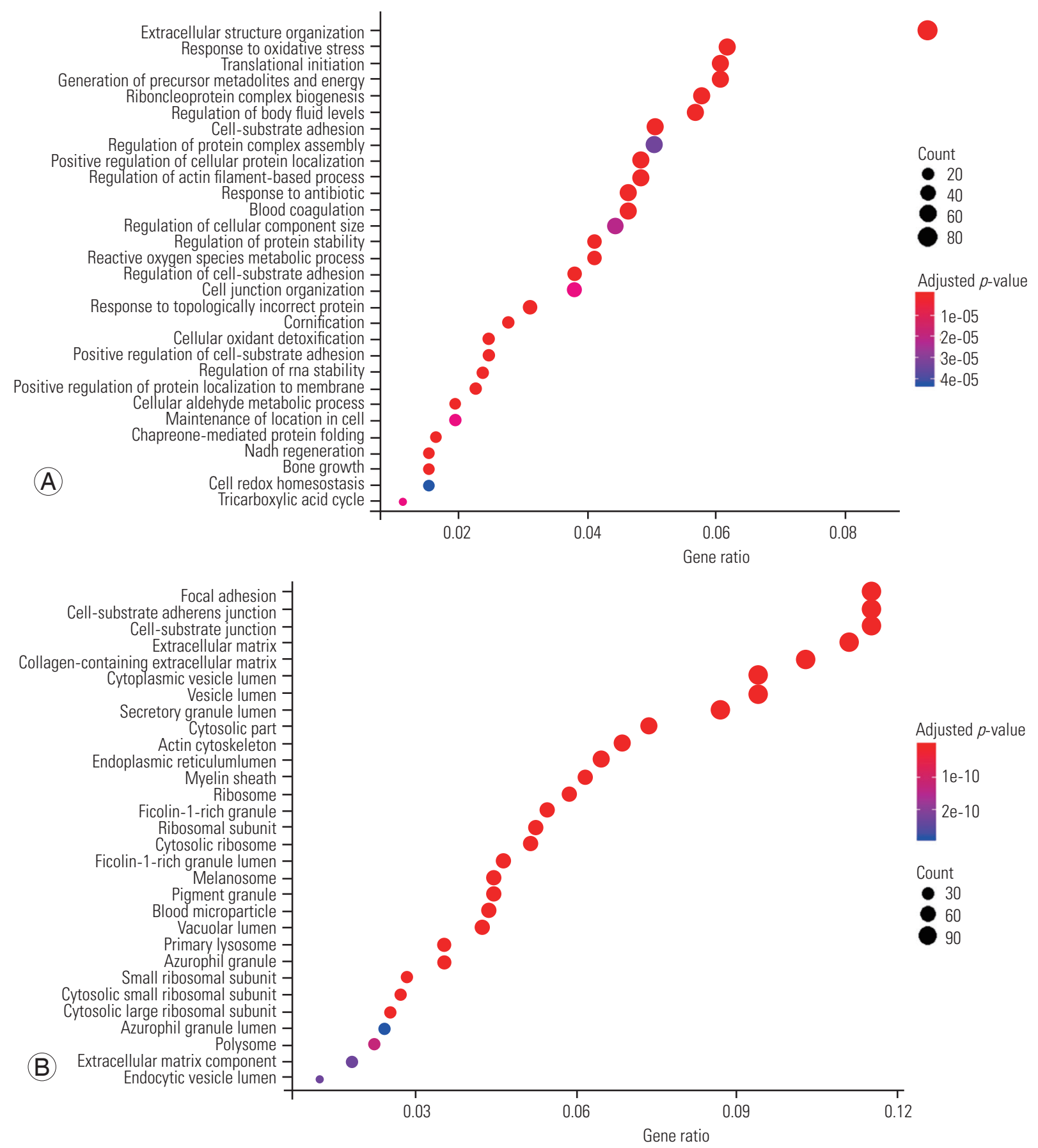

Fig. 4. (A, B) Enriched biological process and localization of proteins in the fetal disc.

considered for further bioinformatics analysis. Clustering using the DAVID Gene Functional Classification Tool identified 10 clusters of highly related proteins (Fig. 5). The topmost cluster had an enrichment score of 17.75 with nine types of collagens. Among the different types of collagens, collagen type XIV alpha 1 chain (COL14A1) was highly expressed, followed by collagen type XI alpha 2 chain, collagen type VI alpha 3 chain, collagen type IX alpha 1 chain, and collagen type VI alpha 1 chain.

The second significant cluster consisted of ribosomal proteins with an enrichment score of 17.131. Among ribosomal proteins, ribosomal protein S16 (RPS16) was expressed nearly 1.5-fold higher than other proteins, followed by ribosomal protein S18 (RPS18), ribosomal protein S7, ribosomal protein S9, ribosomal protein L9, ribosomal protein L23a, and ribosomal protein L27a. 


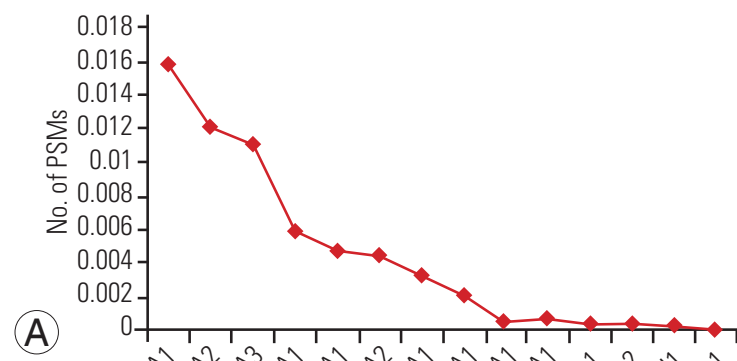

(A)

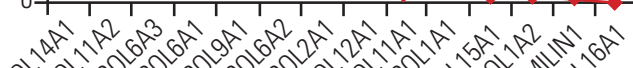

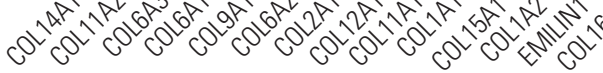
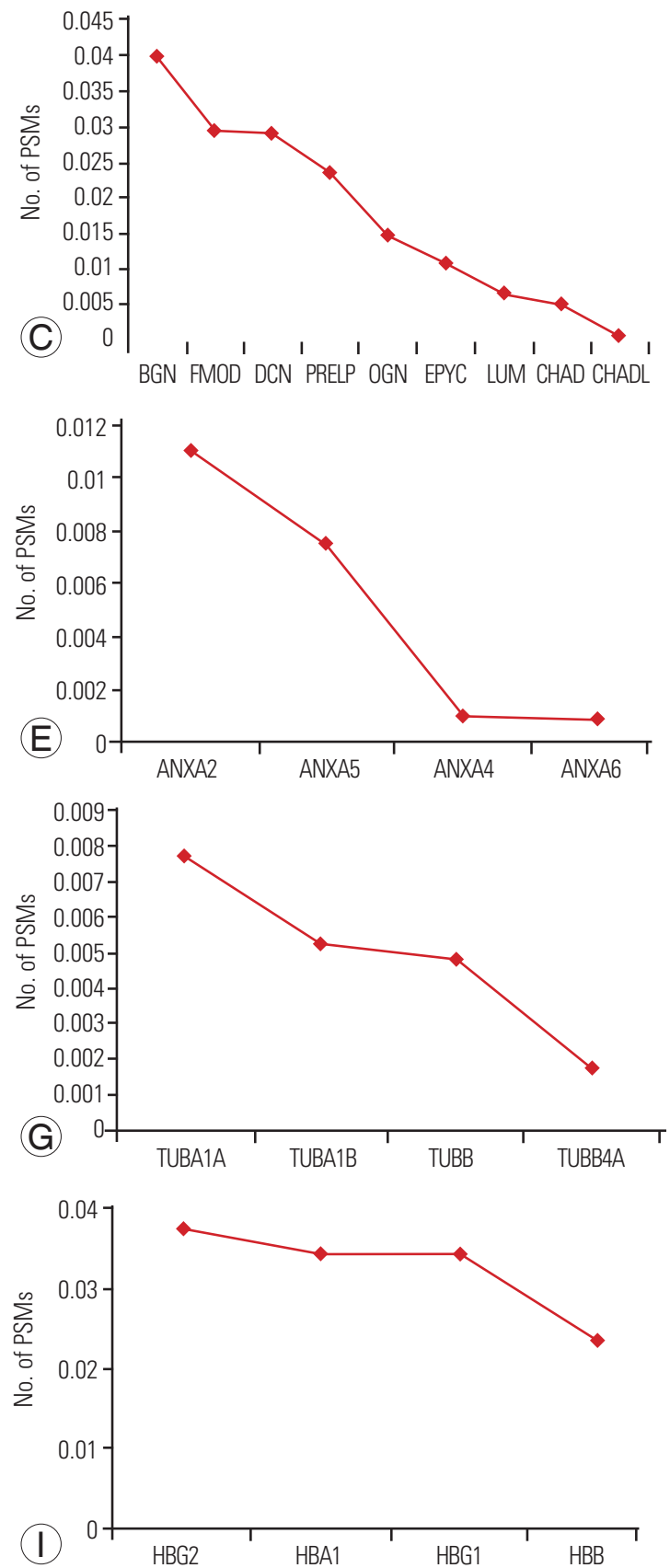
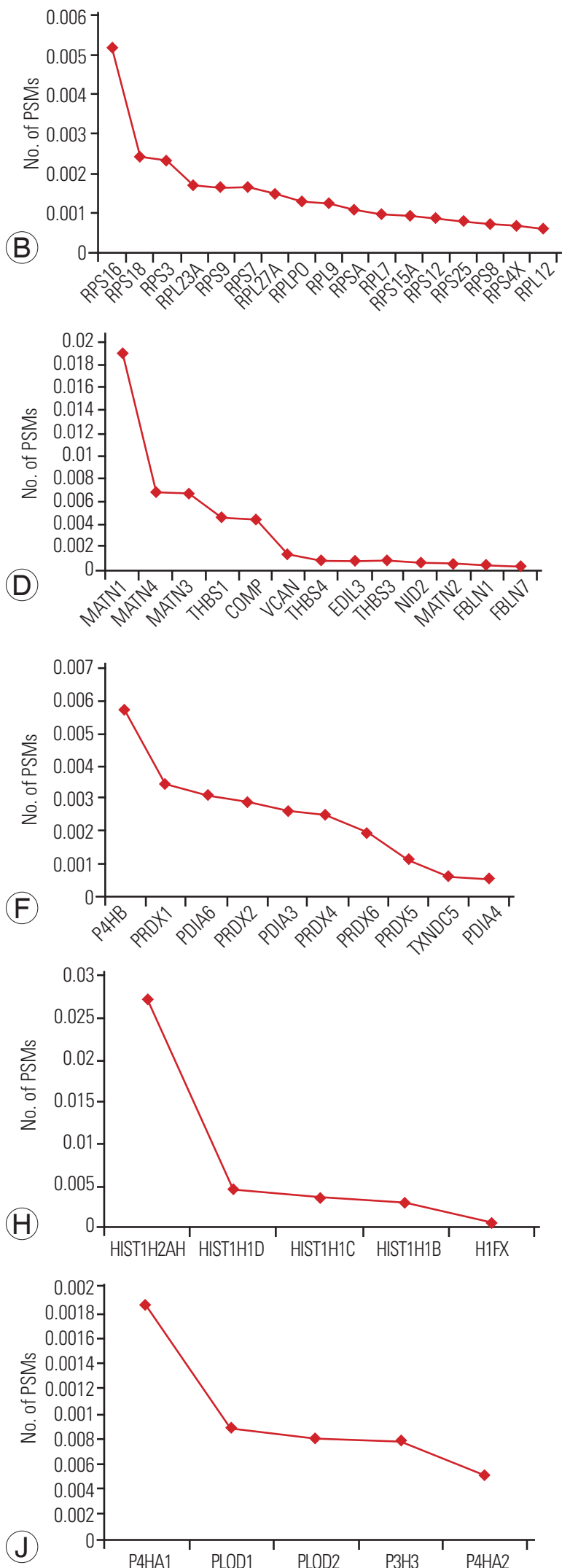

Fig. 5. Graphical representation of fetal protein expression profiles for 10 enriched clusters. (A) Cluster 1: collagens. (B) Cluster 1: ribosomal proteins. (C) Cluster 1: small leucine rich proteins. (D) Cluster 1: matrilin and thrombospondin. (E) Cluster 1: annexins. (F) Cluster 1: protein disulfide isomerase family proteins and peroxiredoxins. (G) Cluster 1: tubulins. (H) Cluster 1: histones. (I) Cluster 1: hemoglobin. (J) Cluster 1: prolyl 4-hydroxylase family proteins. PSMs, peptide spectral matches. 
The third abundant cluster with an enrichment score of 14.77 consisted of SLRPs. It was composed of chondroadherin (CHAD), epiphycan (EPYC), lumican (LUM), biglycan (BGN), fibromodulin (FMOD), proline/arginine-rich end leucine-rich repeat protein (PRELP), chondroadherin like (CHADL), decorin (DCN), and osteoglycin (OGN). BGN, DCN, PRELP, FMOD, and OGN were highly expressed in fetus samples, followed by LUM, CHAD, EPYC, and CHADL.

Interestingly, the fourth cluster of proteins that play a major role in maintaining the extracellular matrix integrity and forming filamentous networks in the extracellular matrices of disc was also identified with an enrichment score of 11.740. It consisted of matrilin 1 (MATN1), matrilin 2, matrilin 3 (MATN3), matrilin 4 (MATN4), thrombospondin 1 (THBS1), thrombospondin 4, thrombospondin 3, fibulin 7, and EGF-like repeats and discoidin domains 3. Among these proteins, MATN1 was found to be highly expressed, followed by MATN3, MATN4, and cartilage oligomeric matrix protein.

A cluster of key enzymes involved in proper threedimensional folding of newly synthesized procollagen chains was also identified with an enrichment score of 11.014. It was composed of prolyl 4-hydroxylase subunit alpha 2, prolyl 3-hydroxylase 3, procollagen-lysine,2-oxoglutarate 5-dioxygenase 2, prolyl 4-hydroxylase subunit alpha 1 , and procollagen-lysine,2-oxoglutarate 5-dioxygenase 1 .

Other than these important clusters, we also identified clusters of peroxiredoxins, protein disulfide isomerase family proteins, annexins, tubulins, histones, and hemoglobin enriched in fetal NP discs.

\section{Pathway analysis}

Pathway enrichment analysis was performed using Reactome database from STRING (Fig. 6). Most of the proteins were found to be involved in extracellular matrix organization ( $p=7.61 \mathrm{E}-26)$, collagen biosynthesis and modifying enzyme pathway ( $p=1.26 \mathrm{E}-21)$, and focal adhesion ( $p=5.85 \mathrm{E}-08)$. Interestingly, we also identified significant pathways involved in the developmental processes. Important pathways such as Hedgehog signaling ( $p=0.0406)$, which is required for the formation of the notochord

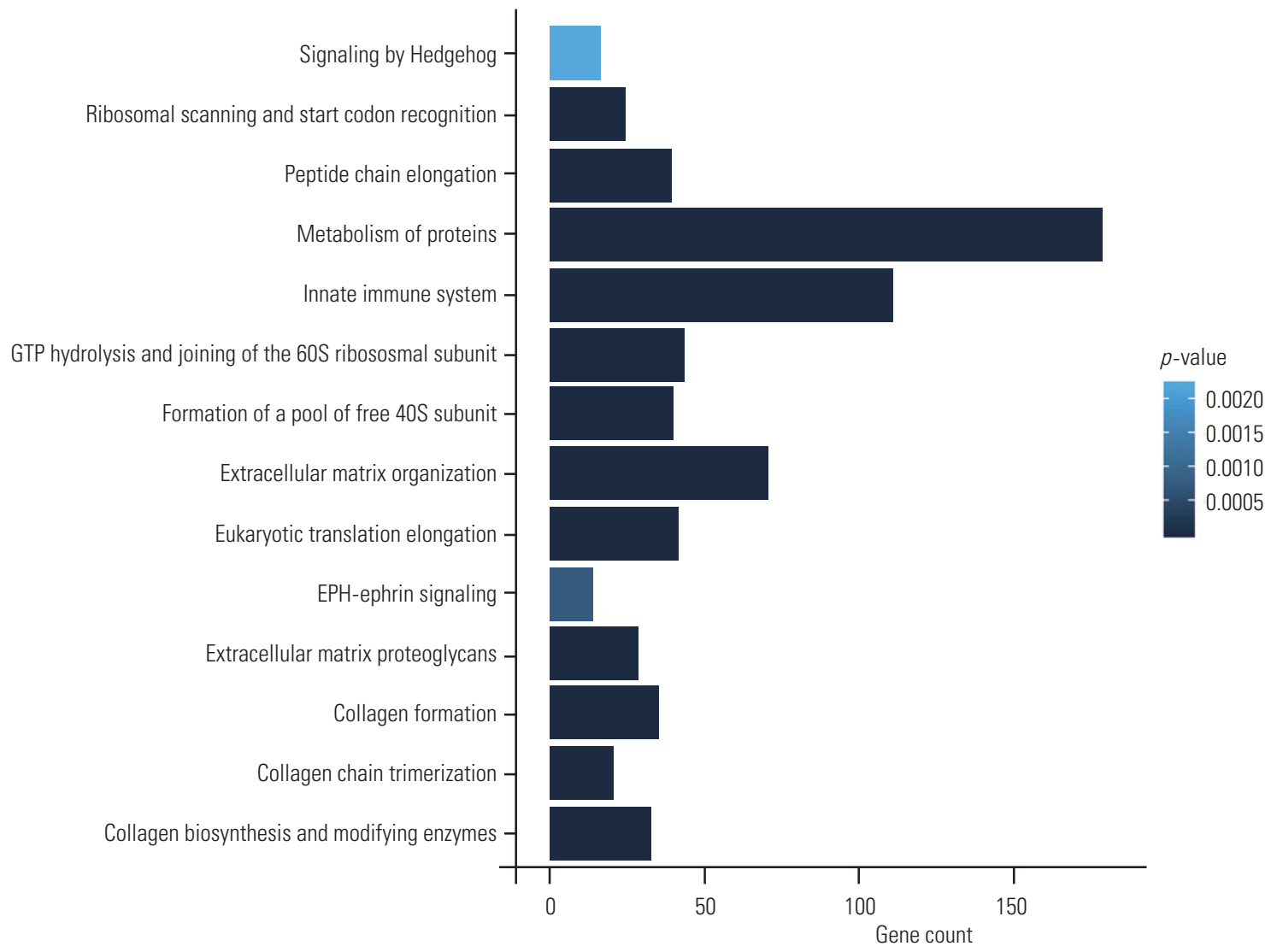

Fig. 6. Enriched pathways in the fetal disc. 
sheath and patterning of NP within the IVDs, were also noted in our samples [3]. We also identified EPH-ephrin signaling pathway $(p=0.0067)$, which is a key regulatory pathway in several important developmental processes such as cardiovascular and skeletal development, tissue patterning, and axon guidance [4].

\section{Discussion}

IVD degeneration, despite being one of the most common causes of low back pain, continues to be the least understood musculoskeletal disorder. Surgical treatments are aimed to alleviate pain and therefore address only the mechanical issues arising from disc degeneration. Among all possible causative factors proposed to be involved in the development of disc degeneration, the inability to maintain matrix homeostasis remains foremost among all possible etiologies. Regenerative molecular therapies have been proposed to trigger disc regeneration, and mesenchymal cell transplantation into IVD was attempted in animal models as early as 2003 [5]. However, further progress could not be made and disc regeneration in humans continues to be challenging due to the inability to replicate native biological environment. It was reported through in vitro culturing of human fetal spine cells and adult NP that fetal spine cells were found to have better matrix synthesizing capacity and hold promise for future cell therapies [6]. However, the key success to such novel therapies will depend on the ability to restore the complex homeostatic mechanisms, which require in-depth understanding of the normal human IVD, including that of a fetus. In this regard, molecular analysis of a healthy disc not exposed to any mechanical and environmental insults may allow us to obtain a clear picture of the physiological, biochemical, and molecular events maintaining disc homeostasis. Recently, gene regulatory networks controlling extracellular matrix synthesis in cervical discs have been identified using RNA sequencing [7]. In this study, efforts were made to document the proteomic signature of normal human lumbar fetal IVDs for the first time, which revealed the molecular and cellular complexities involved in the synthesis of ECM of IVD and also identified molecular targets for further research.

LC-MS/MS analysis of nine fetal IVDs identified a total of 1,316 proteins with the abundant proteins presented in Fig. 3 and Supplemental Table 1. Subsequent GO analysis revealed that fetal disc proteome is significantly enriched with proteins of 14 basic biological processes and five molecular functions, representing disc homeostatic mechanisms in a normal fetal disc (Fig. 4). Of the total proteins, 247 were found in four or more fetal samples, whereas approximately $60 \%$ were found in any one of the fetal samples. This frequency of detection of proteins implies the presence of essential high abundant proteins that dominate spectral features and conceals the low abundant proteins of interest. This may be mainly due to the nature of peptides being hydrophilic or hydrophobic. Hydrophilic or small peptides pass through the reverse-phase column without being ionized, and hydrophobic or large peptides can also be challenging due to poor fragmentation [8]. However, the less frequent or low abundant proteins are more interesting because their modifications or expression levels may carry significant biological information. Moreover, 247 proteins were subjected to cluster analysis, which is a novel method of constructing meaningful sets of similar proteins to analyze their expressions and functions, and this revealed 10 significant clusters of proteins in our study.

The IVD has an outer thick annulus fibrosus, which can withstand tensile forces, and an inner soft NP, which can withstand compressive loads. The NP is derived from the notochord cells in the fetus and has higher cellularity compared to adult discs [9]. The extracellular matrix of the NP primarily consists of two major structural proteins, namely, collagens and proteoglycans. It has been reported that early-stage NP contains abundant collagen II, and aging is associated with an increase in the amount of collagen I [10]. Apart from these, collagen VI and IX have also been reported in NP $[11,12]$. In our proteomic evaluation, collagen was the most significant cluster of proteins as previously reported. Interestingly, apart from collagen I and II, we identified other members of the collagen family, namely, V.VI, IX, XI, XIV, XV, and XVI. Importantly, the most abundant collagen was XIV, followed by XI, VI, IX, XII, II, and others. Collagen XIV is a regulator of fibrillogenesis, and knockout models of this gene in rats have lesser tendinous structures [13]. Previously, this collagen has been reported in the cornea, tendon, articular cartilage, and even skin. However, to our best knowledge, there is no literature on collagen XIV and its role in human IVD. The only previous study indicating its role in the IVD is that on bovine fetus, where its expression was enhanced compared to other collagens [14]. Collagen $\mathrm{XIV}$ is believed to have a major role in disc hydration and 
maintenance of disc height and possibly have a role in regeneration [15]. Abundant expression of collagen XIV in the fetal IVDs signifies its role in the development of IVD and could be an ideal molecule to be targeted for regenerative therapies and tissue engineering techniques.

Ribosomal biogenesis is a basic process occurring in all dividing cells, which involves approximately 80 ribosomal proteins and $>150$ nonribosomal proteins. Ribosomal proteins play an important role in cell growth, proliferation, differentiation, and development [16]. Defects in the genes encoding these proteins have been implicated in human pathologies, such as Diamond-Blackfan anemia and cancer [17]. This highly conserved group of proteins has a $40 \mathrm{~S}$ small subunit (ribosomal protein small subunit [RPS]) and a 60S large subunit (ribosomal protein large subunit $[\mathrm{RPL}]$ ) in humans. In our study, ribosomal proteins formed the second most significant cluster in the fetal proteome. Among the small subunit groups, RPS16, RPS18, and RPS3 and, among the large subunit groups, RPL23, RPL27, and PO were found to be abundant. Ribosomal proteins and their association with disc degeneration have been less investigated. In a previous study, dysregulation of ribosomal genes (RPL8, RPS16, and RPS23) was found to be associated with disc degeneration [18] upon comparison against scoliotic discs. In a recent study on the comparison between degenerated and cadaveric control discs, RPL17, RPL13A, RPL18, and RPS24 were identified among the top 10 central genes of protein-protein interaction network involved in degeneration [19]. In this background, the basal expression of these ribosomal proteins in the fetus from our study reveals that most of them are involved in disc homeostasis and warrants further research on their differential expression during aging and degeneration to explore the possibilities of using them as molecular targets for regenerative therapy.

The third cluster of proteins belongs to the family of SLRPs having five major subclasses and plays an important role in fibrillogenesis, cellular growth, tissue repair, and remodeling, having a potential role in regeneration. In this study, among all proteoglycans, BGN was found to be abundant in the human fetal disc. BGN with DCN interact with other proteins to form a stable extracellular matrix, and BGN-deficient mice have been shown to have accelerated degeneration of IVD due to loss of macromolecular complex assembly [20]. Further, loss of BGN has also been associated with decrease in transforming growth factor- $\beta$, thereby reducing osteoblastic differentia- tion, and this altered bone metabolism might itself result in premature disc degeneration $[21,22]$.

Matrilin and thrombospondin formed the fourth major cluster of proteins. Matrillins belong to a family of oligomeric matrix proteins having a major role in the formation of filamentous networks and maintain the extracellular matrix integrity. In our study, MATN1 was the most abundantly expressed protein and has been found to form a complex with BGN/DCN, which in turn forms a link between aggrecan and collagen VI/II to form a stable extracellular matrix assembly [23]. MATN1 polymorphism and deficiency leading to altered cartilage architecture have been associated with increased incidence and severity of adolescent idiopathic scoliosis [24], and its abundant expression in our fetal disc emphasizes the need for its basal expression to maintain disc structure and homeostasis. It would be interesting to observe their expression in aging and degenerative disc tissues, and if found to be downregulated, it can be used for cell therapy. Similarly, downregulation of THBS-1 (abundantly expressed in the fetus) has been documented to cause progression of disc degeneration [25] and manipulation of either this gene or its product could again be a novel therapeutic strategy.

Overall, the dataset comprising 1,316 different proteins obtained through LC-MS/MS analysis of fetal discs would serve as a molecular repository of IVD and form the basis for studying a series of disc-related diseases like DDD and herniation. Proteomic changes in the IVD across various developmental stages and decades of aging will improve the understanding on the molecular pathology of DDD. For the first time, this study unraveled proteomic consti-

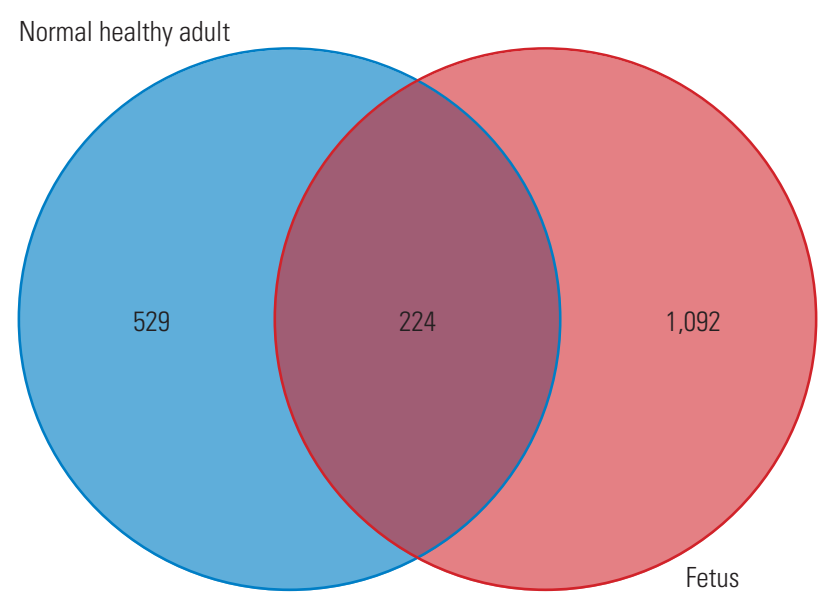

Fig. 7. A Venn diagram comparing the number of proteins extracted from the fetal disc in this study versus adult healthy discs in a previous study showing 1,092 unique proteins in fetal disc samples. 
tution of healthy human fetal disc. A simple comparison of proteins extracted from the fetal disc in this study and human adult disc in a previous study [26], as shown in Fig. 7, reveals that, apart from the 224 common proteins that play a role in disc homeostasis, there are 1,092 proteins in the fetal disc that might have regulatory potential and possibly lead in tissue regeneration therapies. The fetal proteome included a series of proteins involved in extracellular matrix organization. For example, we identified nine types of collagens that are an essential part of the matrix. Collagen fibrils are essential and involved in mechanical strength, cell-fiber interactions, and degradation [27]. Especially, COL14A1, which was highly expressed in fetal disc, plays a major role in fibril assembly. Studies have proved that COL14A1 is a key regulatory molecule in different steps and transitions in fibril assembly and growth during tendon development [28]. Similarly, only key proteins that were most abundantly expressed in the fetal disc have been discussed briefly, as it beyond the scope of including all other molecules mentioned in the results.

\section{Conclusions}

This study provides fundamental information on the proteomic constitution of healthy human fetal disc. A comprehensive catalog of various structural, biochemical, and metabolic regulatory proteins has been established, and this is the first important step in identifying and distinguishing the cellular process and pathways involved in normal biological aging and disease. Proteins expressed most abundantly (COL14A1, BGN, MATN1, and THBS1) in their respective clusters also elucidate the possibility of utilizing these proteins for potential regenerative therapies.

\section{Conflict of Interest}

No potential conflict of interest relevant to this article was reported.

\section{Acknowledgments}

The authors thank Mrs. Sujitha. M of Aravind Medical Research Foundation, for helping us in protein extraction and mass spectrometer analysis.

\section{Funding}

The project was funded by Ganga Orthopaedic Research \& Education Foundation, Coimbatore (Grant no., GOREF-01-2019).

\section{Supplementary Materials}

Supplementary materials can be available from https:// doi.org/10.31616/asj.2019.0217. Supplemental Table 1. Table enlisting the uncharacterized/putative or fragment proteins which were excluded from further bioinformatic analysis.

\section{References}

1. Schizas C, Scaletta C, Burri N, Pioletti D, Applegate. Human adult and fetal intervertebral disc cell cultures: the way forward? Orthop Proc 2006;88-B(Supp_I):152.

2. Zybailov B, Mosley AL, Sardiu ME, Coleman MK, Florens L, Washburn MP. Statistical analysis of membrane proteome expression changes in Saccharomyces cerevisiae. J Proteome Res 2006;5:2339-47.

3. Choi KS, Harfe BD. Hedgehog signaling is required for formation of the notochord sheath and patterning of nuclei pulposi within the intervertebral discs. Proc Natl Acad Sci U S A 2011;108:9484-9.

4. Palmer A, Klein R. Multiple roles of ephrins in morphogenesis, neuronal networking, and brain function. Genes Dev 2003;17:1429-50.

5. Sakai D, Mochida J, Yamamoto Y, et al. Transplantation of mesenchymal stem cells embedded in Atelocollagen gel to the intervertebral disc: a potential therapeutic model for disc degeneration. Biomaterials 2003;24:3531-41.

6. Quintin A, Schizas C, Scaletta C, et al. Isolation and in vitro chondrogenic potential of human foetal spine cells. J Cell Mol Med 2009;13(8B):2559-69.

7. Riester SM, Lin Y, Wang W, et al. RNA sequencing identifies gene regulatory networks controlling extracellular matrix synthesis in intervertebral disk tissues. J Orthop Res 2018;36:1356-69.

8. Karpievitch YV, Polpitiya AD, Anderson GA, Smith RD, Dabney AR. Liquid chromatography mass spectrometry-based proteomics: biological and technological aspects. Ann Appl Stat 2010;4:1797-823. 
9. Risbud MV, Shapiro IM. Notochordal cells in the adult intervertebral disc: new perspective on an old question. Crit Rev Eukaryot Gene Expr 2011;21:2941.

10. Tan CI, Kent GN, Randall AG, Edmondston SJ, Singer KP. Age-related changes in collagen, pyridinoline, and deoxypyridinoline in normal human thoracic intervertebral discs. J Gerontol A Biol Sci Med Sci 2003;58:B387-93.

11. Eyre DR, Matsui Y, Wu JJ. Collagen polymorphisms of the intervertebral disc. Biochem Soc Trans 2002;30(Pt 6):844-8.

12. Wu JJ, Eyre DR, Slayter HS. Type VI collagen of the intervertebral disc: biochemical and electronmicroscopic characterization of the native protein. Biochem J 1987;248:373-81.

13. Ansorge HL, Meng X, Zhang G, et al. Type XIV collagen regulates fibrillogenesis: premature collagen fibril growth and tissue dysfunction in null mice. J Biol Chem 2009;284:8427-38.

14. Caldeira J, Santa C, Osorio H, et al. Matrisome profiling during intervertebral disc development and ageing. Sci Rep 2017;7:11629.

15. Tono-Oka S, Tanase S, Miike T, Tanaka H. Transient expression of collagen type XIV during muscle development and its reappearance after denervation and degeneration. J Histochem Cytochem 1996;44:90718.

16. Zhou X, Liao WJ, Liao JM, Liao P, Lu H. Ribosomal proteins: functions beyond the ribosome. J Mol Cell Biol 2015;7:92-104.

17. Robledo S, Idol RA, Crimmins DL, Ladenson JH, Mason PJ, Bessler M. The role of human ribosomal proteins in the maturation of rRNA and ribosome production. RNA 2008;14:1918-29.

18. Yang Z, Chen X, Zhang Q, et al. Dysregulated COL3A1 and RPL8, RPS16, and RPS23 in disc degeneration revealed by bioinformatics methods. Spine (Phila Pa 1976) 2015;40:E745-51.
19. Mo S, Liu C, Chen L, et al. KEGG-expressed genes and pathways in intervertebral disc degeneration: protocol for a systematic review and data mining. Medicine (Baltimore) 2019;98:e15796.

20. Furukawa T, Ito K, Nuka S, et al. Absence of biglycan accelerates the degenerative process in mouse intervertebral disc. Spine (Phila Pa 1976) 2009;34:E911-7.

21. Xu T, Bianco P, Fisher LW, et al. Targeted disruption of the biglycan gene leads to an osteoporosis-like phenotype in mice. Nat Genet 1998;20:78-82.

22. Harada A, Okuizumi H, Miyagi N, Genda E. Correlation between bone mineral density and intervertebral disc degeneration. Spine (Phila Pa 1976) 1998;23:85762.

23. Wiberg C, Klatt AR, Wagener R, et al. Complexes of matrilin-1 and biglycan or decorin connect collagen VI microfibrils to both collagen II and aggrecan. J Biol Chem 2003;278:37698-704.

24. Burger EL, Noshchenko A, Patel VV, Lindley EM, Bradford AP. Ultrastructure of intervertebral disc and vertebra-disc junctions zones as a link in etiopathogenesis of idiopathic scoliosis. Adv Orthop Surg 2014;2014:850594.

25. Zhu Z, Chen G, Jiao W, et al. Identification of critical genes in nucleus pulposus cells isolated from degenerated intervertebral discs using bioinformatics analysis. Mol Med Rep 2017;16:553-64.

26. Rajasekaran S, Tangavel C, K S SVA, et al. Inflammaging determines health and disease in lumbar discs-evidence from differing proteomic signatures of healthy, aging, and degenerating discs. Spine J 2019 May 22 [Epub]. https://doi.org/10.1016/ j.spinee.2019.04.023.

27. Eyre DR, Wu JJ. Collagen cross-links. In: Brinckmann J, Notbohm H, Muller PK, editos. Collagen. Berlin: Springer; 2005. p. 207-29.

28. Young BB, Gordon MK, Birk DE. Expression of type XIV collagen in developing chicken tendons: association with assembly and growth of collagen fibrils. Dev Dyn 2000;217:430-9. 